\title{
Improving the Accuracy of Radio Astrometry
}

\author{
Alan L. Fey \\ United States Naval Observatory
}

\begin{abstract}
The International Celestial Reference Frame (ICRF) is currently realized by the radio positions of compact extragalactic objects obtained by the technique of Very Long Baseline Interferometry (VLBI). The positions of the defining sources with the highest quality are estimated to be accurate at the 0.25 milliarcsecond (mas) level. The limiting accuracy is thought to be due mostly to tropospheric propagation effects and by the apparent motions of the sources due to variable intrinsic structure. Increasing the accuracy of VLBI astrometry beyond current levels will require an improved understanding of the variable emission structures associated with compact extragalactic sources as well as improvements in the modeling of the Earth's troposphere.
\end{abstract}

\section{Introduction}

The accuracy of current astrometric VLBI is thought to be limited mostly by the apparent motions of the sources due to variable intrinsic structure and by tropospheric propagation effects. This paper reviews a number of analysis improvements which have been successfully employed, or could possibly be used in the future, to increase the accuracy of astrometric observations. It begins with a brief review of the relevance of compact extragalactic sources and the troposphere to astrometric observations.

\subsection{Extragalactic radio sources}

Extragalactic radio sources are assumed to be very distant (typical redshifts of about 1.0) and thus should exhibit little or no detectable proper motions. In the standard theory of extragalactic radio sources (e.g. Blandford and Königl, 1979), emission from quasars and active galactic nuclei is assumed to be powered by a central engine (presumably a black hole) where energetic phenomena occur. The positions on the sky of these engines are thought to be stable at the $\mu$ as level. However, it is well-known that compact extragalactic radio sources observed at VLBI resolutions exhibit variable emission structure on angular scales of order several mas. Extragalactic radio sources are known to have frequency dependent intrinsic structure, usually consisting of a flat spectrum core $(S \propto$ $\left.\nu^{\alpha}, \alpha \approx 0\right)$ with extended emission in the form of multiple steep spectrum $(\alpha \approx$ $-0.5 \sim-1.5$ ) jet components which may move superluminally away from the core (superluminal motion is motion perpendicular to the line of sight with an apparent linear velocity in excess of the speed of light). An obvious consequence is that for extended sources, intrinsic structure will contribute to the uncertainty 
of the measured positions and can introduce systematic position offsets which will appear as absolute motions on the sky. For a more detailed discussion of the contribution of radio source structure to astrometric position determination see Charlot (1990). For more information on the radio structure of a large number of the extragalactic sources which are used for astrometry see Fey \& Charlot $(1997,2000)$.

\subsection{The Earth's troposphere}

Radio signals traveling through the Earth's troposphere (which consists of the lower few tens of kilometers of the atmosphere) experience delay, bending, and attenuation relative to propagation in free-space. Correcting for these effects is a matter of modeling the change in index of refraction (determined mostly by pressure and temperature) along the ray path. The dry component of the troposphere, consisting mostly of $\mathrm{O}_{2}$ and $\mathrm{N}_{2}$, can be considered to be nearly in hydrostatic equilibrium and can thus be be well-modeled by surface meteorology. The water vapor content, on the other hand, is highly variable along the ray path and can undergo large changes and redistribution with changing weather. For a more detailed discussion on modeling tropospheric propagation effects, see the review by Sovers, Fanselow, \& Jacobs (1998).

\section{Improved understanding of source structure variations}

\subsection{Observe at higher radio frequencies}

Due to the nature of compact extragalactic radio sources, one obvious method to improve astrometric observation is to observe at radio frequencies higher than are currently used. Standard VLBI astrometric observations are made at frequencies of $2.3 \mathrm{GHz}$ and $8.4 \mathrm{GHz}$. Dual frequency observations are made in order to be able to remove the extra path length introduced by the Earth's ionosphere. This is standard practice for most astrometric VLBI observations. Possible alternate frequencies could include 15, 22, 43, and perhaps $90 \mathrm{GHz}$.

Benefits An obvious benefit of higher frequency observations is higher angular resolution. For a VLBI interferometer, angular resolution scales approximately as $\lambda / d$, where $\lambda=c / \nu$ is the wavelength of observation and $d$ is the projected baseline length (the projected distance between two radio telescopes as viewed from the source). With current VLBI arrays employing intercontinental baselines, angular resolution approaching $100 \mu \mathrm{as}(\approx 0.4 \mathrm{pc}$ at $z=1)$ is possible at $43 \mathrm{GHz}$. Higher angular resolution is mostly of relevance to imaging observations but has significant consequences for astrometric observations as well. For instance, a VLBI interferometer observing at a frequency of $43 \mathrm{GHz}$ will see less extended jet emission than at $8.4 \mathrm{GHz}$ (the interferometer acts as a spatial filter with less sensitivity to larger scale emission structures at higher frequencies) and will presumably be able to measure the position of the remaining compact core emission more precisely.

Radio source opacity effects should be lessened at higher frequencies. The extended steep spectrum components which show superluminal motion and presumably contribute the most to uncertainties in astrometric position determinations will be weaker and, in the majority of the cases, should be absent al- 
together at the higher frequencies. This has been observed for observations of some sources between $2.3 \mathrm{GHz}$ and $8.4 \mathrm{GHz}$ in the images of Fey \& Charlot $(1997,2000)$. Additionally, because the core in these sources is presumed to be the base of the jet where the optical depth is approximately unity, as shown in the schematic model of a Core-Jet radio source (Figure 2; Fey, these Proceedings), the position of the core should be closer to the center of activity at higher frequencies. Higher frequency observations will measure a more compact source with possibly greater position stability. As an example, the radio structure of the compact extragalactic source $1749+096$ at four frequencies is shown in Figure 1. Note the decrease in the fitted angular size of the source as a function of frequency. The angular size decreases by almost a factor of seven from the lowest to the highest observed frequency.

Finally, because the extra path delay introduced by the Earth's ionosphere scales as the inverse square of the observing frequency, ionospheric effects will be greatly reduced by observing at higher radio frequencies. However, neglecting this dispersion correction altogether would be detrimental to position determination since the interplanetary and interstellar media would still contribute. A "within band" calibration technique at $15 \mathrm{GHz}$ using the National Radio Astronomy Observatory Very Long Baseline Array (VLBA) telescope has been demonstrated (T. M. Eubanks, private communication). This technique makes use of the wide VLBA receiver bandwidth by making near simultaneous dualfrequency observations at 12 and $15 \mathrm{GHz}$ (which are both within the $15 \mathrm{GHz}$ receiver band).

Drawbacks One of the drawbacks of astrometric observations at higher radio frequencies is the possibility of finding fewer suitable sources with sufficient flux density to obtain a reliable position. Observations at $15 \mathrm{GHz}$ and even $22 \mathrm{GHz}$ should encounter little difficulty. However, results of a $90 \mathrm{GHz}$ survey by Lonsdale, Doelman, \& Phillips (1998) detect only $18 \%$ of the sources observed. The low detection rate was attributed to sensitivity levels which were about a factor of two less than are currently achievable. The authors suggest that a detection rate of about $60 \%$ could be obtained in a similar survey with easily attainable sensitivity levels. These results suggest that there will be fewer sources available for astrometric work at higher frequencies.

Decreased sensitivity of the receiving system, as alluded to above, is another problem with astrometric observations at frequencies higher than those currently used. For example, the sensitivity of the VLBA at $15 \mathrm{GHz}$ is about $24 \%$ less than at $8.4 \mathrm{GHz}$. At $22 \mathrm{GHz}$, the VLBA is about $58 \%$ less sensitive and is about $72 \%$ less sensitive at $43 \mathrm{GHz}$. Loss of sensitivity leads to decreased signal-to-noise ratio (SNR) and hence larger position uncertainties.

Propagation effects due to turbulence in the troposphere is enhanced at higher frequencies as the wavelength of observation approaches the turbulence scale length. The result is decreased phase coherence time necessitating shorter duration observations with the consequence of lower SNR and hence larger position uncertainties.

Finally, most radio telescopes used for astrometry and geodesy are designed to operate optimally at the standard radio frequencies of 2.3 and $8.4 \mathrm{GHz}$. Upgrading these telescopes for higher frequency observations would be a costly venture. 

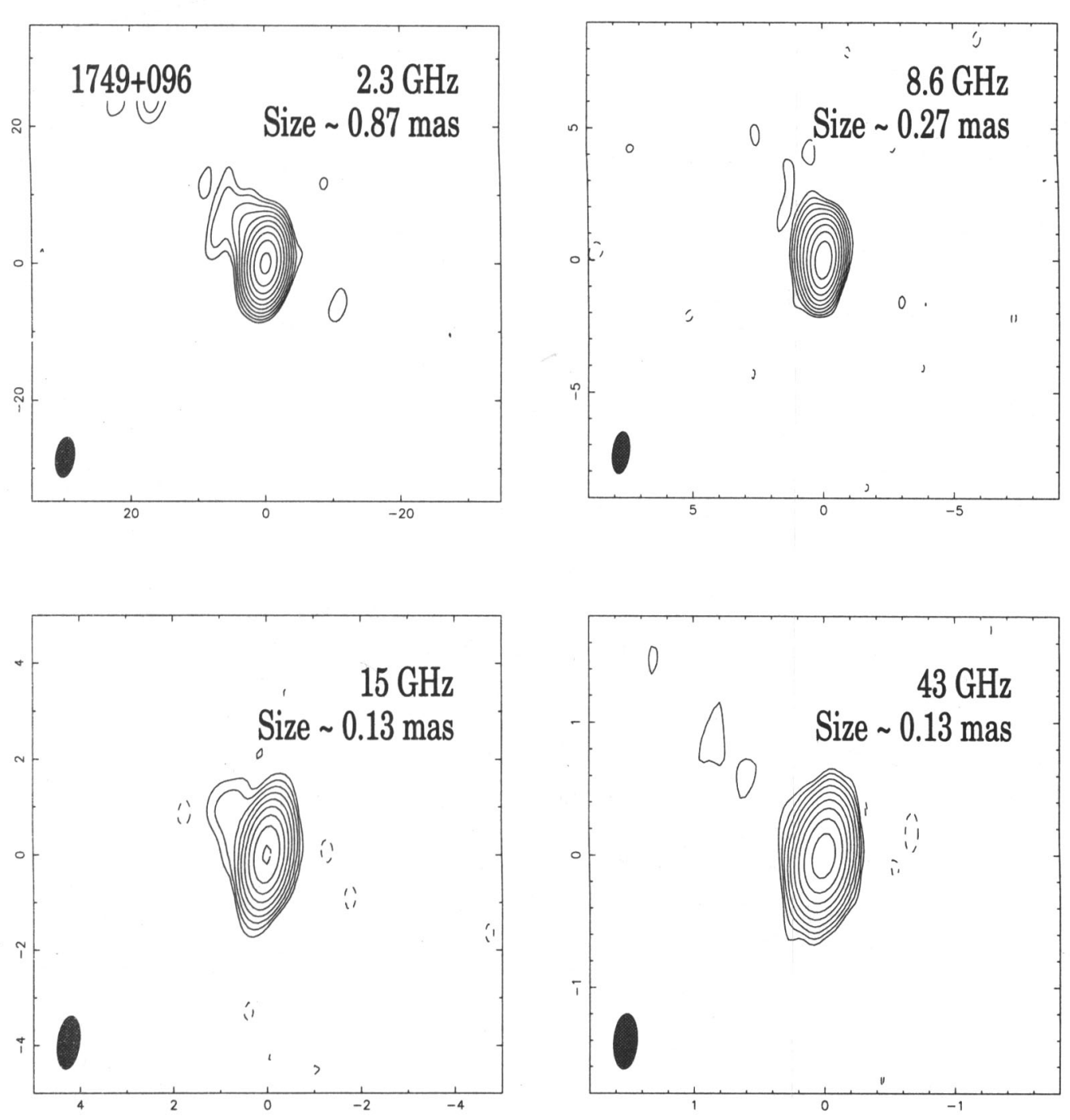

Figure 1. The radio frequency structure of the compact extragalactic source $1749+096$ at four frequencies. The angular size (full width at half maximum) of a circular Gaussian component fitted to the data, together with the frequency of observation, is listed in each panel. Note the change in angular scale of the images from the lowest to the highest frequency. 


\subsection{Structure monitoring}

Maintenance of the ICRF at a high level of accuracy requires measuring and monitoring changes in source structure in order to determine both the short and the long-term effects of variable intrinsic source structure on astrometric position determination. To this end, the U.S. Naval Observatory has initiated and is continuing an observing program to image the radio reference frame sources on a regular basis at the same radio frequencies used for precise astrometry ${ }^{1}$. These data have allowed a number of investigations into the effects of intrinsic radio source structure on astrometric position determination (Fey \& Charlot, 1997; 2000; Charlot, these Proceedings).

\subsection{Structure index}

As shown by Charlot (1990), the contribution of intrinsic source structure to a VLBI bandwidth synthesis delay measurement can be significant and depends on the exact form of the spatial brightness distribution of the extended radio source relative to the geometry of the VLBI baseline vector projected onto the plane of the sky. Fey \& Charlot $(1997,2000)$ used VLBA images of 388 ICRF sources to calculate structure corrections to the bandwidth synthesis delay and then divided the sources into four categories, according to the calculated structure corrections, by defining a source "structure index." The best sources were given a structure index of 1 , while the worst sources were given a structure index of 4 . Two structure indices were obtained for each source, one at $2.3 \mathrm{GHz}$ and one at $8.4 \mathrm{GHz}$, each of which provides an indication of the source structure effect magnitude at the corresponding frequency band.

\subsection{Source structure delay corrections}

Inclusion of radio source intrinsic structure information into the astrometric solutions from which the radio positions are derived is another option for increasing the astrometric quality of the sources. Significant progress has been made in this area (Charlot, these Proceedings) but this is an ongoing work and has not yet been fully demonstrated.

\section{Improved modeling of the troposphere}

\subsection{New mapping functions}

A mapping function is defined as the ratio of the electrical path length along a ray to a source to the electrical path length along a ray at the zenith. A new mapping function developed by Niell (2000) using in-situ data from numerical weather prediction or analysis has been successfully demonstrated to decrease the errors associated with determining the dry component of the troposphere by about a factor of two at an elevation of $5^{\circ}$ over previous mapping functions. The wet component is improved by only about $25 \%$ at $5^{\circ}$ elevation.

\footnotetext{
${ }^{1}$ Data obtained to date are available for scientific use by anyone and can be accessed from the United States Naval Observatory's Radio Reference Frame Image Database at http: // wwr.usno.navy.mil/RRFID/.
} 


\subsection{Gradients}

The use of a non-azimuthally symmetric troposphere model (gradient) removed a systematic effect in the ICRF source positions (Ma et al., 1998) that had a maximum magnitude on the order of 0.5 mas. The effect is much larger than the formal errors of the positions and is caused by the greater troposphere thickness near the Earth's equator (MacMillan \& Ma, 1997).

\subsection{Water vapor radiometers}

Determining the water vapor content of the troposphere along the observing direction continues to be one of the most elusive aspects of calibrating astrometric observations. One solution would be to use a water vapor radiometer (WVR). A WVR measures the water vapor content along the line of site, which can be used to determine the wet troposphere delay. The Haystack Observatory (http://ww.haystack.mit.edu/) has successfully developed an inexpensive WVR for use with their Coordinated Millimeter VLBI Array program. The Haystack WVR has been shown to have a sensitivity of $\approx 0.4 \mathrm{~mm}$ of $\mathrm{H}_{2} \mathrm{O}$ path length in a one-second integration.

\section{The Mark-IV VLBI system}

The new Mark-IV VLBI system, which is currently coming into wide-spread use, potentially has increased sensitivity by about 2-3 times that of its predecessor, the Mark-III system. Improved sensitivity leads to higher precision astrometric measurements with more rapid sky coverage using shorter duration observations.

\section{The future}

In the next decade, there will be significant advances in the area of spacebased optical astrometry. Proposed and scheduled missions such as the National Aeronautics and Space Administration's Space Interferometry Mission (SIM), the U.S. Naval Observatory's (USNO) Full-Sky Astrometric Mapping Explorer (FAME), and the European Space Agency's Global Astrometric Interferometer for Astrophysics (GAIA) mission will achieve positional accuracies well beyond that presently obtained by any ground-based radio interferometric measurements.

In order to remain competitive with these new space-based optical missions, one possibility to improve the accuracy of VLBI astrometry would be to place a radio interferometer in space. The concept and utility of placing a radio telescope in Earth orbit has been demonstrated by the Japanese HALCA satellite (http://www.vsop.isas.ac.jp/). An obvious benefit of space-based VLBI would be the absence of propagation effects due to the troposphere and the ionosphere - the only remaining major source of uncertainty would be from the variable intrinsic structure of the sources. Observations at radio frequencies higher than are currently used, coupled with structure modeling, could be used to minimize the effects of source structure. However, the feasibility of radio astrometry in space is completely unknown and remains for future astrometrists. 


\section{References}

Blandford, R. D. \& Königl, A., 1979, Astrophys. J., 232, 34.

Charlot, P., 1990, Astron. J., 89, 1309.

Fey, A. L. \& Charlot, P., 1997, Astrophys. J. Sup., 111, 95.

Fey, A. L. \& Charlot, P., 2000, Astrophys. J. Sup., in press.

Lonsdale, C. J., Doelman, S. S., \& Phillips, R. B., 1998 Astron. J., 116, 8.

Ma, C. et al., 1998, Astron. J., 116, 516.

MacMillan, D. S. \& Ma, C., 1997, Geophys. Res. Lett., 24, 453.

Niell, A., 2000, in Proceedings of the First IVS General Meeting, N. R. Vandenberg (ed), NASA/CP-2000-209893.

Sovers, O., Fanselow, J., \& Jacobs, C., 1998, Reviews of Modern Physics, 70, p. 1393. 